\title{
Determination of diallyldimethylammonium chloride in drinking water by reversed-phase ion-pair chromatography-electrospray ionization mass spectrometry
}

\author{
Fen Jin ${ }^{\mathrm{a}, \mathrm{b}}$, Jianying Hu ${ }^{\mathrm{b}, *}$, Min Yang ${ }^{\mathrm{a}}$, Xiaohui Jin ${ }^{\mathrm{b}}$, Wenjie $\mathrm{He}^{\mathrm{c}}$, Hongda Han ${ }^{\mathrm{c}}$ \\ a State Key Lab of Environmental Aquatic Chemistry, Research Center for Eco-Environmental Sciences, Chinese Academy of Sciences, Beijing 100085, China \\ ${ }^{\mathrm{b}}$ College of Environmental Science, Peking University, Beijing 100871, China \\ ${ }^{\mathrm{c}}$ Tianjin Waterworks Company Ltd., Tianjin 300040, China
}

Received 10 June 2005; received in revised form 10 October 2005; accepted 10 October 2005

Available online 21 October 2005

\begin{abstract}
A method for the direct determination of diallyldimethylammonium chloride (DADMAC) in water samples, using ion-pair liquid chromatography-mass spectrometry system was developed. The chromatographic separation was performed using a C18 column. The type, the concentration of ion-pair reagent and the $\mathrm{pH}$ were optimized to give good chromatographic retention and sensitivity for DADMAC. Quantification was achieved in the positive electrospray ionization mode using selected ion monitoring. The cone voltage was also studied to establish the optimal experimental conditions. Finally, the reproducibility of the proposed method was shown by good run-to-run and day-to-day precision values. No sample preparation was required and the detection limit was $0.1 \mu \mathrm{g} / \mathrm{L}$. The method was used to detect residual DADMAC at drinking water treatment plants in Tianjin, north China. The concentration of DADMAC observed in drinking water ranged from below quantitation limit to $22.0 \mu \mathrm{g} / \mathrm{L}$.
\end{abstract}

(C) 2005 Elsevier B.V. All rights reserved.

Keywords: Diallyldimethylammonium chloride; Drinking water; Ion-pair chromatography; LC-ESI- MS

\section{Introduction}

Synthetic polyelectrolytes are widely used in drinking water treatment, either as flocculants in conjunction with a metal salt coagulant or, less commonly, as coagulants in their own right. Poly-diallyldimethylammonium chloride (PDADMAC) is a water-soluble cationic polymer, which is widely used as a coagulant in potable water treatment for the last thirty years [1-3]. The maximum dose of PDADMAC used in drinking water treatment process has been regulated in Canada and the USA, and its residual concentration in drinking water has been regulated to be $50 \mu \mathrm{g} / \mathrm{L}$ in the USA [4]. However, there is no regulation of the monomer, diallyldimethylammonium chloride (DADMAC, Fig. 1), which is thought to be released and become a potential contaminant in drinking water. Although no peer-reviewed literature reported the toxicity data about

\footnotetext{
* Corresponding author. Tel.: +86 1062765520 ; fax: +86 1062765520 .

E-mail address: hujy@urban.pku.edu.cn (J. Hu).
}

DADMAC, a positive result for the genotoxicity of DADAMC was found using the SOS/umu test in our lab in unpublished data. To assess the risk of DADMAC, the need for sensitive identification of DADMAC in environmental water has increased.

To the best of our knowledge, no studies on the analysis and occurrence of DADMAC in environmental and drinking water have been reported in the literature. Several analytical methods for cationic surfactants and quaternary ammonium compounds (QACs), have been developed for water quality investigation in water treatment industries. These methods typically use the complex reaction between QACs and anionic dye, which can be measured colorimetrically [5]. However, they are unsuitable for monitoring DADMAC in drinking water due to the trace levels. The reversed-phase ion-pair chromatography-electrospray ionization mass spectrometry technique has been successfully applied to analyze quaternary ammonium compounds in water, such as diquat, mepiquat and paraquat [6-12]. The method of liquid chromatography-electrospray ionization mass spectrometry (LC-ESI-MS) allows for unequivocal identification of the 
<smiles>CCCC1C[N+](C)(C)CC1CCC</smiles>

PDADMAC

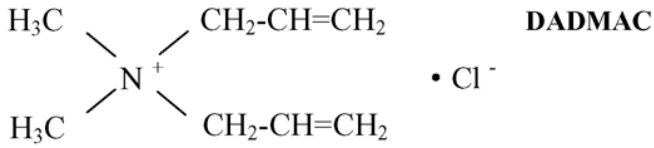

Fig. 1. Structures of PDADMAC and its monomer (DADMAC). $n$ : the number of monomers of DADMAC.

compound in drinking water samples because of its specificity and sensitivity.

In this study, we developed a reversed-phase ion-pair chromatography-mass spectrometry method for identifying trace DADMAC in drinking water. To the best of our knowledge, this is the first description of a methodology for quantitative analyses of DADMAC and the first instance where DADMAC has been observed in drinking water.

\section{Experimental}

\subsection{Reagents and materials}

An aqueous DADMAC solution $(60 \%(\mathrm{w} / \mathrm{v}, \%))$ was obtained from Tokyo Chemical Industry (Tokyo, Japan). The exact content of DADMAC was determined to be $62.99 \%$ (w/v) by weighing the weight after freeze-drying the solution of $1.000 \mathrm{~mL}$. Heptafluorobutyric acid (HFBA) was obtained from Sigma-Aldrich (Steinheim, Germany). Trifluoroacetic acid (TFA) was from Acros (New Jersey, USA). ASC-grade dimethyl sulfoxide (DMSO) was obtained from Amresco (Solon, USA). HPLCgrade acetonitrile and methanol were purchased from Fisher (New Jersey, USA). Ultra-pure water was prepared using an Easypure UV Compact Ultrapure System (Fisher Chemical, China) under a resistivity of $18.2 \mathrm{M} \Omega / \mathrm{cm}$. Formic acid and ammonium formate were analytical grade obtained in China. The $\mathrm{pH}$ meter was from Leici (Shanghai, China). Stock standard solution of DADMAC $(3.150 \mathrm{mg} / \mathrm{mL})$ was prepared in water and the working solutions were obtained by serially dilution with water. All solutions were stored at $4{ }^{\circ} \mathrm{C}$.

\subsection{Sample collection}

Sampling was generally carried out at raw water and drinking water from a drinking water treatment plant in Tianjin, north China. Luhe River $(888 \mathrm{~km})$ is the source of drinking water for the Tianjin area. Water samples were collected in polyethylene bottles prior to filtering through a $0.45 \mu \mathrm{m}$ filter (Whatman $\mathrm{GF} / \mathrm{F}$, Whatman, Maidstone, UK). Before tap water samples were stored, $\mathrm{Na}_{2} \mathrm{~S}_{2} \mathrm{O}_{3}(1 \mathrm{~mol} / \mathrm{L})$ was added to decompose the residual $\mathrm{HOCl}$. To avoid adsorption, all samples were stored in disposable, precleaned polyethylene bottles at $4{ }^{\circ} \mathrm{C}$ until analysis. And all samples should be analyzed as soon as possible.

\subsection{LC-ESI-MS analysis}

LC-ESI-MS, in positive ion mode, was used to analyze the DADMAC in the water samples. The analyte was separated by using a Waters 2695 HPLC (Milford, USA) equipped with a quaternary gradient pump, column oven, and an autosampler with a $100 \mu \mathrm{L}$ injection loop. The analytical column was an XTerra C18 column $(150 \mathrm{~mm} \times 2.1 \mathrm{~mm}, 3.5 \mu \mathrm{m}$; Waters, USA). Column temperature was maintained at $50{ }^{\circ} \mathrm{C}$. The mobile phase used for eluting the analyte from the HPLC column consisted of acetonitrile and $5 \mathrm{mM}$ HFBA aqueous solution ( $\mathrm{pH} 4.2)$, at a flow rate of $0.2 \mathrm{~mL} / \mathrm{min}$. A gradient elution was performed as a linear gradient from $99.5 \% \mathrm{~A}(5 \mathrm{mM} \mathrm{HFBA}, \mathrm{pH} 4.2)$ and $0.5 \%$ $\mathrm{B}$ (acetonitrile) to $60 \% \mathrm{~A}$ and $40 \% \mathrm{~B}$ in $10 \mathrm{~min}$ and then held isocratic at $60 \% \mathrm{~A}$ for $2 \mathrm{~min}$, followed by a stepwise elution carried out from $60 \% \mathrm{~A}$ and $40 \% \mathrm{~B}$ to $0 \% \mathrm{~A}$ and $100 \% \mathrm{~B}$. The injection volume was $20 \mu \mathrm{L}$. The HPLC system was connected to a platform ZQ single quadrupole mass spectrometer (Micromass, Manchester, UK) equipped with an ESI probe operated in positive ionization mode. The orthogonal Z-Spray interface allowed the entire column effluent from the LC to be directed into the source without flow splitting, and contributed to the greatly enhanced sensitivity. In the positive mode, typical ion source parameters were used as follows: ESI capillary voltage at $2.8 \mathrm{kV}$; extractor voltage at $4 \mathrm{~V}$; source block temperature at $130{ }^{\circ} \mathrm{C}$; desolvation temperature at $400{ }^{\circ} \mathrm{C}$; ion energy at $0.8 \mathrm{~V}$; multiplier voltage at $650 \mathrm{~V}$. Nitrogen was used as the desolvation gas with a flow rate of about $440 \mathrm{~L} / \mathrm{h}$ and as the cone gas with a rate of $60 \mathrm{~L} / \mathrm{h}$; the cone voltage was ramped from 20 to $60 \mathrm{~V}$ with the full scan mass ranging from 50 to $500 \mathrm{Da}$ with a scan time of $0.5 \mathrm{~s}$. The $\mathrm{M}^{+}$ion $(\mathrm{m} / \mathrm{z}, 126)$ for DADMAC was used in selected ion monitoring (SIM) mode, with a dwell time of $100 \mathrm{~ms}$ and inter channel time of $1 \mathrm{~ms}$.

\section{Results and discussion}

\subsection{Effect of cone voltage on the fragmentation of $D A D M A C$}

Based on the fact that the cone voltage is one of the most important characteristics influencing fragmentation in LC-ESIMS, its effects on the fragmentation and sensitivity of DADMAC were investigated. Fig. 2 shows LC-ESI-MS mass spectra for DADMAC by direct injection at different cone voltages. Cone voltages at 20, 40 and $60 \mathrm{~V}$ were applied to study the main fragment ions formed under LC-ESI-MS. At the low cone voltage of $20 \mathrm{~V}$, the compound produced the molecular ion $\mathrm{M}^{+}(\mathrm{m} / \mathrm{z}$ 126) as a base peak, and a fragment ion $\left[\mathrm{M}-\mathrm{C}_{3} \mathrm{H}_{6}\right]^{+}(\mathrm{m} / \mathrm{z}$ 84) with $10 \%$ relative intensity which resulted from the breaking of the carbon-nitrogen bond between the quaternary amine and the propylene substituent. At a cone voltage of $40 \mathrm{~V}$, the fragment ion $\left[\mathrm{M}-\mathrm{C}_{3} \mathrm{H}_{6}\right]^{+}$was the base peak in the mass spectrum of DADMAC and the relative intensity of $\mathrm{M}^{+}$was reduced to $10 \%$. Substantial fragmentation occurred at $60 \mathrm{~V}$ and the $[\mathrm{M}]^{+}$ion $(\mathrm{m} / \mathrm{z}$ 126) was completely fragmentized. At a cone voltage of $60 \mathrm{~V}$, the fragment ion $\left[\mathrm{M}-\mathrm{C}_{3} \mathrm{H}_{6}-\mathrm{C}_{2} \mathrm{H}_{3}\right]^{+}$was the base peak in the mass spectrum of DADMAC. As expected, higher cone voltage 

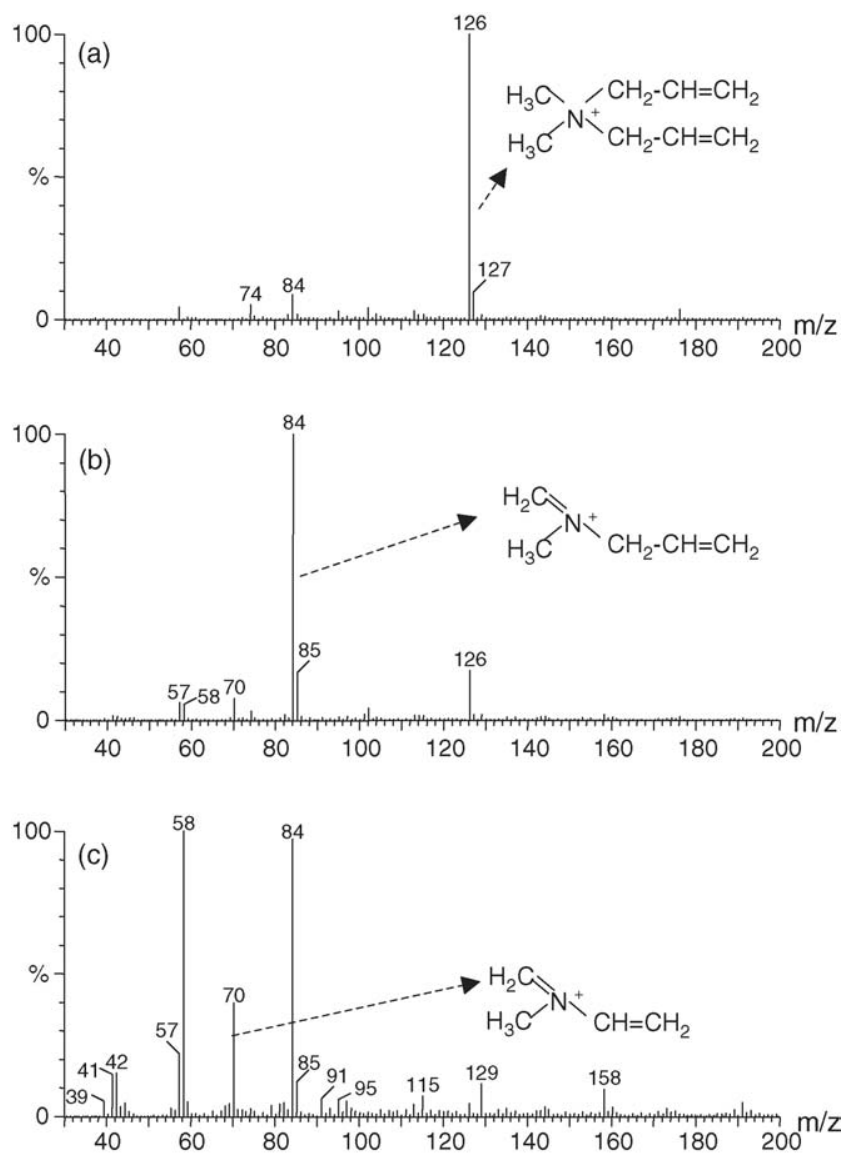

Fig. 2. LC-ESI-MS mass spectra for DADMAC $(157.4 \mu \mathrm{g} / \mathrm{L})$ in positive ion mode. Cone voltage: (a) $20 \mathrm{~V}$; (b) $40 \mathrm{~V}$ and (c) $60 \mathrm{~V}$.

leads to decrease of abundance of the molecular ion and intensive fragmentation. Although the substantial structural information was at $60 \mathrm{~V}$, the highest sensitivity occurred at $20 \mathrm{~V}$, which was four times higher than the sensitivity at $60 \mathrm{~V}$. Considering the probable trace level in the environment, all samples were subsequently analyzed with multi-channel mode with quantification performed at $20 \mathrm{~V}$ to ascertain the sensitivity and qualitative analysis performed at $60 \mathrm{~V}$ simultaneously.

\subsection{Optimization of the ion-pair chromatograph}

Ion-pair chromatographic separation by using a C18 reversed-phase was achieved for DADMAC. In this study, in order to suppress the broad and tailing of peak due to the silanol interaction with DADMAC, the mobile phase was adjusted to $\mathrm{pH} 4.2$ resulting in narrower and symmetrical peaks. However, a very low retention was observed using the Xterra C18 column mainly due to the DADMAC ionic character. An attempt to improve the retention of DADMAC was made by adding ionpair reagents such as HFBA and TFA to the mobile phase. The retention time and the relative responses of DADMAC had a significant difference when using the two ion-pair reagents at the same concentration $(5 \mathrm{mM})$, as shown in Fig. 3a. As expected, HFBA produced the better sensitivity than TFA, which was similar to the results reported in previous paper [13]. From the view of retention on the column and analytical sensitivity for DAD-

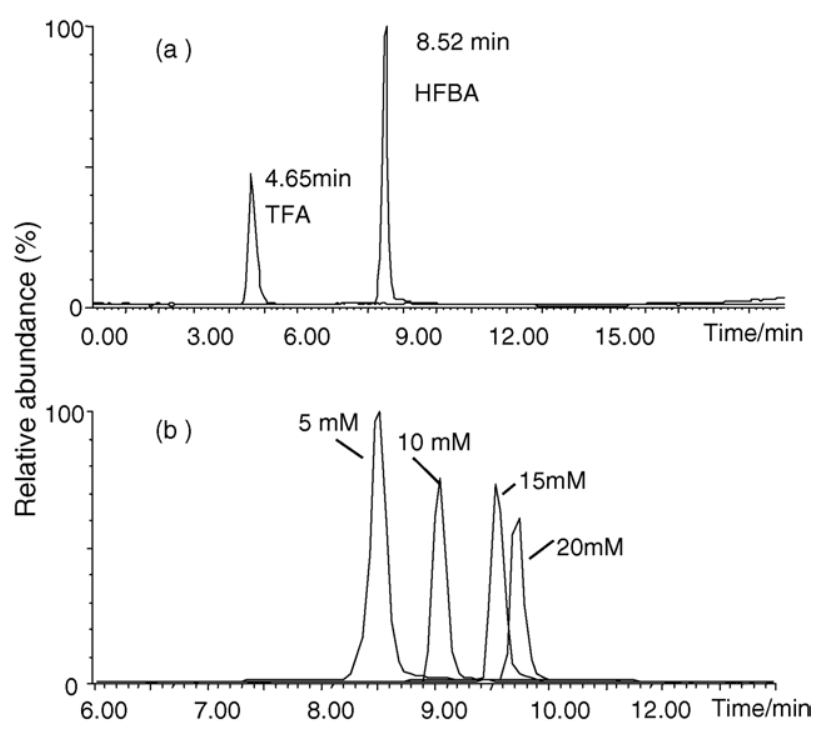

Fig. 3. LC-ESI-MS chromatograms (positive ion mode) of a standard solution of DADMAC (157.4 $\mu \mathrm{g} / \mathrm{L})$ : (a) effects of ion-pair reagents and (b) effects of HFBA concentration. Selected ion: $126(\mathrm{~m} / \mathrm{z})$.

MAC, HFBA was chosen. The effects of HFBA concentration on the retention and response were also studied by changing the concentration from 5 to $20 \mathrm{mM}$. Fig. $3 \mathrm{~b}$ shows the chromatograms obtained at different HFBA concentrations. While the retention increased with increasing the amount of HFBA from 5 to $20 \mathrm{mM}$, the ESI signal for DADMAC was found to be suppressed, a phenomenon which was also found by Gustavsson et al. in their analysis of eight amine analytes [14]. As a result, $5 \mathrm{mM}$ HFBA aqueous solution ( $\mathrm{pH} 4.2$ ) was used as the mobile phase in this study.

\subsection{Accuracy, precision and detection limit for $L C-M S$}

Precision, linearity and limits of detection were determined using standard aqueous solutions. Calibration curves were constructed for the standard solutions between 0.1 and $157.4 \mu \mathrm{g} / \mathrm{L}$, and good linearity was observed $\left(r^{2}>0.99\right)$, indicating good performance of the methodology developed in this work. Six replicate determinations of $15.74 \mu \mathrm{g} / \mathrm{L}$ standard aqueous solutions were carried out on the same day under the optimum conditions to determine the run-to-run precision of LC-MS analysis using the ESI ionization technique. The relative standard deviation (RSD) was 6.6\%. DADMAC is a cationic, and can be adsorbed on the walls of the sample container. Polyethylene containers were tested in this study. The drinking water sample spiked with DADMAC at levels of $15.74 \mu \mathrm{g} / \mathrm{L}$ was analyzed. During 15 days, there was no obvious variation in the concentration. The RSD was $12 \%$ by day-by-day replicate determinations, indicating good repeatability of the method developed in this work. The detection limit was estimated to be $0.1 \mu \mathrm{g} / \mathrm{L}$ based on a signal-noise ratio of 3:1 using standard solutions in the SIM mode. The above result suggests the method established in this study has the capability of selectively detecting low concentrations of DADMAC in environmental and DWTP water samples. 
Table 1

Concentrations $(\mu \mathrm{g} / \mathrm{L})$ of DADMAC in water samples from Tianjin Drinking Water Treatment Plant

\begin{tabular}{lll}
\hline Sampling date & Raw water & Drinking water \\
\hline $04 / 21 / 2003$ & nd & nd \\
$05 / 25 / 2003$ & nd & nd \\
$07 / 03 / 2003$ & nd & 5.4 \\
$08 / 28 / 2003$ & nd & 11.7 \\
$09 / 26 / 2003$ & nd & 17.8 \\
$04 / 20 / 2004$ & nd & nd \\
$06 / 20 / 2004$ & nd & 15.7 \\
$08 / 24 / 2004$ & nd & 22.0 \\
\hline
\end{tabular}

Note: nd, not detected (below quantitation limited).

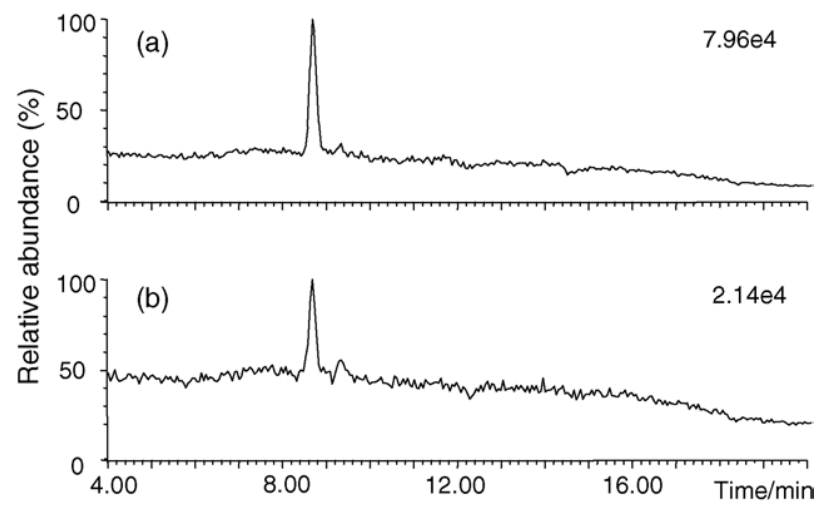

Fig. 4. LC-ESI-MS chromatograms of DADMAC in (a) drinking water on July 2003; and (b) diluted commercial PDADMAC solution $(45 \mu \mathrm{g} / \mathrm{L})$. Selected ion: $126(\mathrm{~m} / \mathrm{z})$.

\subsection{Environmental application}

Finally, the ion-pair LC-ESI-MS method established in this study was applied to analyze the residual DADMAC in raw water and drinking water. Raw water samples and drinking water samples taken from the Drinking Water Treatment Plant in Tianjin, north China, were analyzed to investigate the occurrence of the target components from 2003 to 2004 (Table 1). It was found that while DADMAC was detected in five drinking water samples of which concentration ranged from 5.4 to $22.0 \mu \mathrm{g} / \mathrm{L}$, DADMAC was below quantitation limit in all raw water samples. Typical LC-ESI-MS chromatograms for drinking water are shown in Fig. $4 \mathrm{a}$, the concentration was $5.4 \mu \mathrm{g} / \mathrm{L}$ on July 2003 . The higher concentrations of DADMAC in drinking water occurred in the summer, and the concentration was ranged from below quantitation limit to $22.0 \mu \mathrm{g} / \mathrm{L}$. In addition, it should be noted that no DADMAC was detected in raw water, suggesting that the DADMAC in drinking water would stem from the drinking water treatment process.
Moreover, the residual amounts of DADMAC in commercial PDADMAC made in China were analyzed by the method developed in this study. The commercial PDADMAC (Tianjin, China) is a slurry containing $38 \%(\mathrm{w} / \mathrm{v})$ PDADMAC. After diluting the concentration of PDADMAC to $45 \mu \mathrm{g} / \mathrm{L}$ from the commercial PDADMAC, DADMAC was detected to be $2.0 \mu \mathrm{g} / \mathrm{L}$ (Fig. 4b). Thus, the percentage of DADMAC was estimated to be $4 \%$ by weight on an active polymer of this commercial product. This abundance of DADMAC in PDADMAC is higher than the lowest level of monomeric DADMAC (1\%) that can be achieved with the current, best-available manufacturing technology.

\section{Conclusions}

An ion-pair LC-ESI-MS method with high sensitivity and specificity was established for analyzing DADMAC in environmental water and drinking water by adapting a $\mathrm{C} 18$ column with acetonitrile/water ( $\mathrm{pH} 4.2)$ for chromatographic separation. The DADMAC concentration detected in drinking water shows that more attention should be pay to its residue in drinking water.

\section{Acknowledgements}

Financial support by State High Tech. Research and Development (863) Project of China [2002AA601140-6] and the National Natural Science Foundation of China [40021101] is gratefully acknowledged.

\section{References}

[1] C. Wandrey, J. Hernandez-Barajas, D. Hunkeler, Adv. Polym. Sci. 145 (1999) 123.

[2] B.A. Bolto, Progr. Polym. Sci. 20 (1995) 987.

[3] G.L. Amy, P.A. Chadik, J. Am. Water Works Assoc. 75 (1983) 527.

[4] NSF (National Sanitation Foundation, International), Drinking water treatment chemicals-Health effects; Document NSF/ANSI 60-2002, New York, 2002.

[5] T. Sakai, N. Ohno, Talanta 33 (1986) 415.

[6] R. Castro, E. Moyano, M.T. Galceran, J. Chromatogr. A 830 (1999) 145.

[7] Y.V. Taguchi, W.D.S. Jenkins, W.P. Crozier, D.T. Wang, J. Am. Soc. Mass. Spectrom. 9 (1998) 830

[8] R. Castro, E. Moyano, M.T. Galceran, J. Chromatogr. A 914 (2001) 111.

[9] K. Kummerer, A. Eitel, U. Braun, J. Chromatogr. A 774 (1997) 281.

[10] O. Nunez, E. Moyano, M.T. Galceran, Anal. Chim. Acta 525 (2004) 183.

[11] P. Fernandez, A.C. Alder, M.J.F. Suter, W. Giger, Anal. Chem. 68 (1996) 921.

[12] I. Ferrer, E.T. Furlong, Environ. Sci. Technol. 35 (2001) 2583.

[13] T.M. Annesley, Clin. Chem. 49 (2003) 1041.

[14] S.A. Gustavsson, J. Samskog, K.E. Markides, B. Langstrom, J. Chromatogr. A 937 (2001) 41. 